\title{
Cell Origin of Tumors and the Persistence of Cancer Propagating Cells in Tumor Lesions
}

\author{
Donatella Aldinucci ${ }^{*}, 1, \S$ and Roberto Perris ${ }^{1,2, \S}$ \\ ${ }^{I}$ Experimental Oncology 2, Centro di Riferimento Oncologico, I.R.C.C.S., Aviano (PN), Italy \\ ${ }^{2}$ Department of Evolutionary and Functional Biology, University of Parma, PARMA, Italy
}

\begin{abstract}
Cells defined as "cancer stem cells" because of a postulated ability to self-renew in a stem cell-like fashion and regenerate a tumor analogous to that of provenience upon transplantation have been described in a range of myeloid leukemia, breast, prostate, brain, colon and ovarian cancers. However, the precise origin of these cells remains an enigma. Surgical pathologists have suspected for decades that connective tissue tumors could arise through the neoplastic transformation of an immature multipotent cell and that this cell persisted in the lesion in a transformed state. This hypothesis was later supported by some experimental data generated by hematologists who demonstrated the presence of stem/progenitor-type cells in different types of leukemia. In fact, increasing evidence now suggests that quiescent leukemic cells resembling normal hematopoietic stem cells and displaying a clonogenic self-renewal capacity are central in sustaining these pathologies. Although it remains technically challenging to verify the precise origin of a tumor, recent experimental findings in animal models incite a revisiting of the dogma that epithelial tumors may solely form as a consequence of a multistep "dedifferentiation process" of specialized epithelial cells. Moreover, despite the fact that progressive accumulation of genetic aberrations may remain the primary event leading to malignant transformation, some gastrointestinal tumors have been proposed to originate from stem cells residing within discrete niches of the intestinal crypta. Thus, the recent identification of discrete populations of tumor initiating cells in a number of carcinomas, melanomas and brain tumors, may be interpreted as to assign a more predominat role than previously thought to immature progenitor cells just as the ones specifically targeted by neoplastic transformation.
\end{abstract}

\section{WHY WOULD IMMATURE PROGENITOR CELLS ORIGINATE TUMORS?}

Conceivably, the delicate cell-cycle regulation of stem cells, and the fact that these cells divide asymmetrically, may afford a rational explanation for their increased susceptibility to the genetic hits that are known to be instrumental for the conversion of a healthy cell into a cancer cell. Furthermore, several studies have now documented the ability of healthy multipotent progenitor cells to be transformed into neoplastic cells by genetic manipulations mimicking those that may occur spontaneously in tissues during tumorigenesis (Fig. 1A,B) [1]. Subpopulations of cells within tumor lesions that have recently been proposed to constitute the cellular core that assures maintenance and propagation of the tumor, and which are at the focus of attention for therapeutic intervention, may correspond to the foci of cells that originally underwent transformation. Therefore, it could be predicted that a better definition of the genetic, molecular and behavioral traits of the currently denoted "cancer stem cells" may favor the assertion of their incipient nature and their putative derivation from a counterpart healthy cell. Accordingly, the present article discusses the current experimental findings that point to the possibility that particularly immature, and possibly pluripotent, progenitor cells may originate various cancer types. However, we also highlight some of the misconceptions revolving around the notion of "cancer stem cells" that,

*Address correspondence to this author at the Experimental Oncology 2, Centro di Riferimento Oncologico, IRCCS, via F: Gallini 2, Aviano I33081, Italy; Tel: 00-0434-659413; Fax: 00-0434-659428;

E-mail: daldinucci@cro.it

${ }^{\S}$ Both authors contributed equally to this work. in the absence of crucial pieces of evidence, simply identify a subset of particularly malignant, largely drug-resistant cells within a tumor lesion. The importance of defining the cellular identity of tumor origin by clonogenic means is certainly one of the keys to better understand how tumors evolve and progress to more aggressive phenotypes, as well as to establish the nature of the putative residual cells within cancer lesions that may possibly have retained stem/progenitor celllike properties.

\section{APPROPRIATENESS OF ADOPTING THE DEFINI- TION "CANCER STEM CELL" TO DENOTE THE MOST AGGRESSIVE POPULATION OF THE LE- SION}

Subsets of malignant cells within cancer lesions seem to fulfil a similar task as normal stem cells, i.e. to repair/reconstitute a tissue/lesion in which they reside. This fact has incited investigators to adopt the term "cancer stem cells" to denote such a neoplastic cell subpopulation [2-4]. The idea is that the three most distinctive properties of normal stem cells may partly be exhibited by the presumptive "cancer stem cells": 1) the capacity of self-renewal (i.e. proliferate in an alternated manner symmetrically or asymmetrically without undergoing any differentiation or maturation process and without loosing capacity to further replicate), which allows maintenance of the undifferentiated stem cell pool over the lifetime of the organism; 2) "self-regulate" the stem cell population number through balancing self-renewal against differentiation of the daughter cells; and 3) undergo a sufficient degree of differentiation and maturation to allow for the reconstitution and long-term preservation of the 

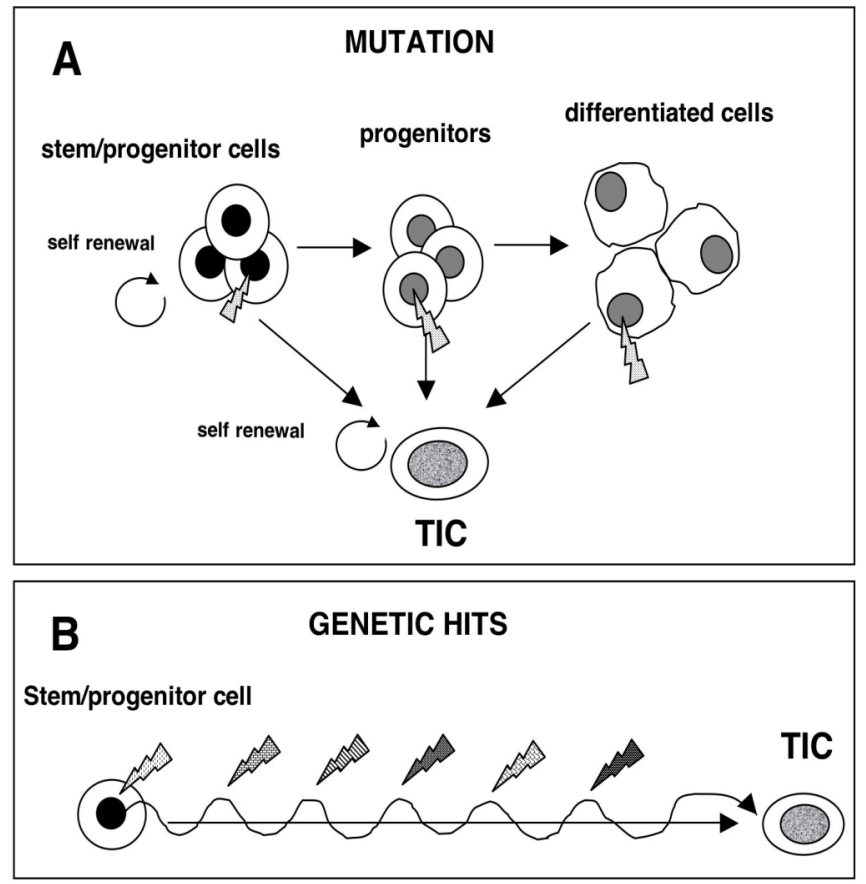

\section{MULTISTEP DEDIFFERENTIATION}
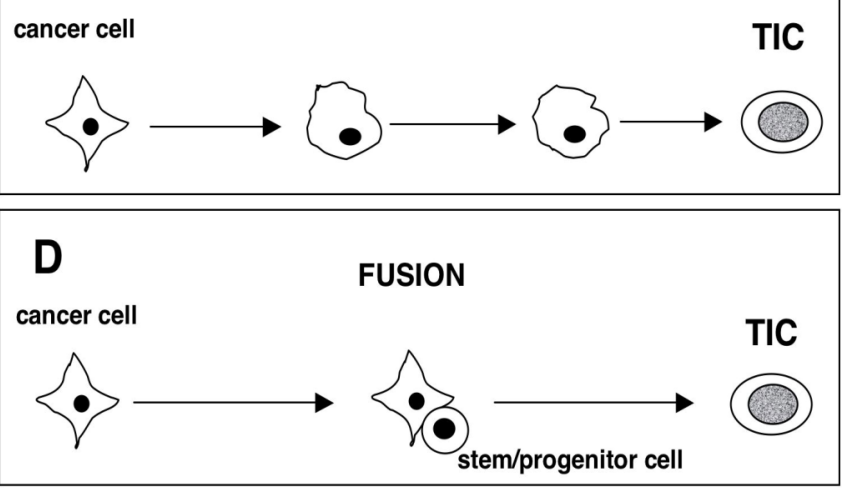

Fig. (1). Potential mechanisms of TIC formation. (A) Mutation. The cancer stem cells might appear after mutations in specific stem cells or early stem cells progenitors. It is also possible that TIC can be derived from differentiated cells. (B) Multiple genetic hits. Progressive genetic alterations drive the transformation of stem/progenitor cells into TIC. (C) Multistep de-differentiation. Multistep dedifferentiation of cancer cells might give rise to TIC. (D) Cell fusion. Cell fusion between cancer cells and stem/progenitor cells might induce TIC.

functional elements (cell lineages) of a tissue. The extent to which these specific features of normal stem cells may actually be preserved in "cancer stem cells" remains to be established. More importantly, with "cancer stem cells" one may merely refer to a small subgroup of cells within a tumor that is particularly dispensable for perpetuating its growth. These cells, better defined as "tumour initiating cells" (TIC), could be the source of all the malignant cells in a primary tumor, they could compose the small reservoir of drug-resistant cells that are responsible for relapse after a therapy-induced remission, or they could be elements of a primitive lesion more directly implicated in formation of metastases. No incontrovertible evidence is presently available to fully substantiate or reject any of these possibilities, whereas indis- putable is the fact that rare sets of cells that firmly diverge from the others are contained in most tumor lesions [5-17].

\section{POSSIBLE ORIGIN OF TIC}

Tumor initiation and progression is generally thought to occur as a multistep process characterized by progressive genetic alterations that drive the transformation of normal human cells into highly malignant derivatives $[18,19]$. This idea has led to the belief that most cancers arise from a single cell that undergoes a malignant conversion driven by frequent genetic mutations. Such events are thought to be followed by a clonal selection of variant cells that show increasingly more aggressive behavior. The hypothesis has been put forward that some of the cellular and molecular events leading to tumor initiation are orchestrated by the subset of cells that could correspond to the ones postulated to display self-renewal capacities (Fig. 1B) [20].

There is compelling evidence in support of the ancient idea that mesenchymal tumors arise by transformation of an immature "fibrohistocytic cell", derived from studies showing that pleomorphic sarcoma cells share their phenotype with bone marrow-derived mesenchymal progenitor cells [21]. These same cells can be converted into Ewing's sarcoma-like cells by the simple transfer of the oncogenic fusion product (EWS-FLI-I) characterizing this specific tumour [22]. Although not yet explored, a similar result would likely be obtained if analogous oncogenic fusion transcripts (e.g. the liposarcoma FUS-DDIT3 or the synovial sarcoma SYT-SSX1) would be transfected into the above cells. Of note, however, is that the bone marrow-derived cells used in these studies are not yet ascertained to be bona fide stem cells, but may rather be multipotent progenitor cells with a limited self-renewal capability. The possibility that these cells may acquire a full auto-regenerating capacity upon neoplastic transformation and thereby, become truly selfrenewing in their transformed state, is an alluring hypothesis worth addressing. The above findings would nonetheless still be perfectly coherent with the fact that many sarcoma lesions contain neoplastic cells exhibiting multiple phenotypic traits. They are also compatible with the fact that osteosarcomas, showing a typical osteoblastic phenotype, are frequently encountered in subcutaneous locations where bone-forming cells would not be present, but where mesenchymalfibroblastic cells are known to reside. It could still be argued that a specific derivation from immature cells could be proper of a mesenchymal tumor, but not of epithelial-derived ones, i.e. tumor that arise within epithelial tissues and exhibit phenotypic traits compatible with epithelial cells. However, at least one investigation convincingly shows that even epithelial tumors may arise from cells of non-epithelial origin [23]. Whether this implies that all epithelial cells have the same formation dynamics remains highly uncertain. On the other hand, stem/progenitor have been identified in virtually all primary epithelial tissue types/organs and may potentially be prone to undergo neoplastic transformation (Fig. 1A,B).

Some brain tumours seem to contain neural precursorlike cells responding to the same mitogenic stimuli as normal neural stem cells and apparently mimicking some of their behavior. Subsets of these brain tumor cells also share crucial markers with normal stem cells, such as CD133, and 
efficiently grow in sphere-like arrangements (so called neurospheres) just as normal neural stem cells. By exploiting the CD133 surface molecule for immunoaffinity purification, several groups have been able to isolate putative tumor initiating cells (TIC) from glioblastoma multiform and medulloblastoma tumors and have proposed that clonogenic cell populations derived from these cells can reproduce the same type of nervous tumors in vivo upon transplantation into mice [5-7]. These observations strongly suggest that neural stem/progenitor cells undergoing neoplastic transformation may correspond to the TIC generating the lesion. On the contrary, the reason $\mathrm{CD} 133^{+}$subset of brain tumor cells would posses self-renewing capability seems unclear, simply on the basis of the assumption that $\mathrm{CD}_{133^{-}}$are not tumorigenic and that cells of the $\mathrm{CD} 133^{+}$population generate single cell clones resembling the parental cells. Multipotency observed in cell populations isolated and further selected by low density plating of cells disaggregated from brain tumors and cultured under serum-free conditions in the presence of EGF/FGF-2 strongly support the idea that neoplastic cells, with similar differentiation potential as neural stem cells isolated from the foetal brain, are present within glioblastoma multiforme lesions. Whether these cells alone and directly initiated the lesion, or whether they were generated secondarily by de-differentiation of more mature neural cells that underwent neoplastic transformation, remains to be defined (Fig. 1C).

While the presence of $\mathrm{CD} 133^{+}$cells with tumor initiating capability may have a strong connection with the normal stem cells of that organ, the reason for which colon carcinoma TIC should share the above membrane marker $[15,16]$ is much less clear. CD133 was first identified on subsets of haematopoietic progenitor cells, later found to be assimilated to circulating endothelial precursors [24], and owing to the recognized analogies between haematopoietic and nervous systems the coincidence of this phenotypic marker could be predicted for both normal and cancer-related progenitor-type cells. On the other hand, CD133 has also been identified in a number of human adult tissues, most prominently in the kidney, and may in all these locations characterize immature progenitor cells [25]. Thus, two scenarios may be contemplated: colon cancer $\mathrm{CD} 133^{+}$TIC may correspond to endogenous immature cells of the colon that have undergone transformation locally, or they may represent a precursor population recruited to the tumor lesion via circulation that, in the new neoplastic microenvironment, has undergone changes. At present, the former possibility seems the most likely considering that colon adenocarcinoma cell lines may express CD133 and modulate their expression under the influence of differentiation-promoting stimuli [25].

Most cancers are not clonal, but consist of heterogeneous cell populations, and using some imagination these have been compared to the hierarchical tree of stem cell-derived phenotypic lineages [26-28]. In such a scheme, it would be attractive to think about a long-lived tumour cell at the base of the tree because it would readily explain how progression towards malignancy could rapidly be achieved through an amplification of mutations and epigenetic changes through the various levels. As alluded above, many normal cells, both in vitro and in vivo, can become cancerous after transduction with the right combination of oncogenes, but it seems that only certain cells are prone to undergo such trans- formation (Fig. 1A,B). Whether these are cells predisposed to become the subset of cells that will constitute the final tumor initiating cell population is a matter of debate.

\section{DO CELL FUSION, EPIGENESIS AND MICROENVI- RONMENT CONTROLLING THE INCEPTION OF TIC?}

Somatic stem cell plasticity has been challenged by observations indicating that apparent transdifferentiation of bone marrow-derived stem cells invading other tissues/organs might be caused by cellular fusion between stem cells and pre-existing differentiated cells. Since current models of carcinogenesis based on the gene mutation hypothesis have limitations in explaining many aspects of cancer, new models of multistage carcinogenesis have been proposed, including the idea that cancer development involves both gene mutations and cell fusions. Specifically, a tumor could result from fusion between an "altered" pre-malignant cell and a stem/progenitor cell resident of that tissue/organ (Fig. 1D). "Aneuploidy", which is a hallmark of malignancy [19] is a direct consequence of such cell fusion and the alternative model of tumor initiation might be the fusion of stem cells with cells that have undergone a set of mutational events related to cancer development. Such fused cells would probably express stem/progenitor cell features and might show large chromosomal aberrations and aneuploidy. They could also harbor unique cell-survival programs that are shared by normal stem cells and that might drive tumor progression. Therefore, a stem/progenitor cell fused to a somatic cell that has received a number of defined mutational hits might explain the presence of the chromosomal derangements that can occur during early tumor development. Fusion might also occur between different tumor cells and, although likely a rare event, it may still have relevance for tumor progression.

A recent hypothesis is that cancer has a fundamentally common basis that is grounded in a polyclonal epigenetic disruption of stem/progenitor cells, mediated by "tumorinducing genes". Accordingly, Feinberg and collaborators have proposed that non-neoplastic, but epigenetically disrupted stem/progenitor cells, may be a key target for tumor formation [29]. Pathological epigenetic changes (nonsequence-based alterations that are inherited through cell division) are increasingly considered as alternatives to mutations and chromosomal alterations in disrupting gene function. These include global DNA hypomethylation and hypermethylation of selected key genes, aberrant chromatin modifications and loss of imprinting. All of these epigenetic changes have the potential to lead to aberrant activation of growth-promoting genes and aberrant silencing of tumorsuppressor genes. Furthermore, tumor cell heterogeneity is due in part to epigenetic variation in progenitor cells, and epigenetic plasticity together with genetic lesions drives tumor progression. This crucial early role for epigenetic alterations in cancer is in addition to genetic alterations that can substitute for genetic variation later in tumor progression. A second step involves monoclonal genetic mutation of gatekeeper genes (or characteristic chromosomal rearrangements in leukaemia or lymphoma), followed by a third step that involves acquisition of genetic and epigenetic plasticity. The above paradigm includes a key step formerly commonly recognized as neoplasia, which can help to explain the late on- 
set of most adult cancers, recurrent disease, environmental effects, tumor heterogeneity and the genetics of cancer risk that may be directly or indirectly associated with TIC.

In the adult organism, stem cells reside in a physiologically limited and specialized microenvironment, or niche, that supports stem cells but varies in nature and location depending on the tissue type [30]. The stem cell niche is believed to be represented by a group of cells and their surrounding extra-cellular matrix (ECM) that is delimited to special locations within tissues and serves the purpose of sustaining stem cells and keeping them in an undifferentiated state. The niche largely works as a physical anchoring site for stem cells and, accordingly, a number of adhesion molecules have been implicated in the interaction between stem cells and niche components. In addition, the niche comprises numerous factors, including various growth factors, cytokines and signalling molecules such as hedgehogs, Wnt:s and BMP:s, that participate in the control of stem cell proliferation and fate determination. In the haematopoietic, intestinal, and hair follicle systems, the niches seem to further maintain stem cells in a quiescent state by providing signals that inhibit cell proliferation. Therefore, stem cell propagation may depend upon dynamic responses of the cells to nicheassociated signals, where the balance between mitogenic signals and growth arresting/differentiation signals is pivotal in their homeostatic regulation. If this balance is disrupted, stem cells may proliferate without restraint, such as occurring upon loss of BMP signalling or abnormal activation of Wnt signalling, and may be engaged in tumorigenic processes [31]. However, recent evidence has suggested that the stem cell niche may have a counteracting function that has thus far been underestimated, namely to prevent tumorigenesis by controlling stem cell proliferation [32]. The niche aspect nevertheless remains crucial since recent investigations have commenced to address the possibility that TIC may also reside within specific niches [33] and it may be speculated that if these sites are truly the preferred locations of TIC they may represent the original territories of tumor formation.

\section{EVIDENCE FOR TIC IN TUMORS AND CHARAC- TERISTICS OF THE COMPRISED CELLS}

The notion of the existence of a specific cell subset within solid tumor lesions endowed with self-renewal properties, has stemmed from the operational/experimental observation that not all cells of a given tumor formation are capable of generating a second tumor mass, upon transplantation. This irrespectively of whether it is done in a heterologous or autologous context. However, a systematic analysis of the tumor generating capability of single cancer cells, or their clonal derivations in a healthy host, has not yet been performed with a sufficient amount of randomly selected cells/clones from a human or animal tumor lesion. Thus, a tight correlation between the diversity of cancer cells composing a lesion and their ability to regenerate a tumor in vivo still need to be more clearly established. The fact that a variable amount of cells are needed to generate a (second) tumor lesion when transplanted into an unconditioned recipient may reflect the need for a substantial cell-to-cell interaction to create a growing mass, or to the fact that, irrespectively of a lack of immunological attack (i.e. in severely immunocompromised animals), many transplanted cells succumb to the trauma of transplantation and/or a defective microenvironmental stimulation. In fact, it is not clear to what extent tumor cells within a transplanted population, compete for host microenvironmental factors. On the other hand, in syngenic immunocompetent settings, as little as 50-100 unselected cells of a particularly aggressive cell line (such as e.g. B16 melanoma cells) are sufficient to generate a significant tumor mass.

At present, there is no evidence that cell lines notoriously behaving in a highly aggressive manner have a constitutive higher ratio of TIC and, hence, a simple hypothesis of TIC based on such quantitative "transplantation parameters" does not warrant full support. The term "cancer stem cell/TIC" has largely been adopted as an operational term defined by the ability of a population of cancer cells, mostly selected on the basis of given cell surface marker profiles (Table 1) to regenerate a similar tumor for an indefinite number of times (although practically most researchers have fixed four times as the "magic number") when transplanted into a host. Once again, however, this feature has not been demonstrated at the single cell level (as has been done with normal haematopoietic cells) [34] leaving the nature and intrinsic properties of the postulated self-regenerating cells contained by these transplantable tumor cell populations undefined, as well as their effectiveness in generating a tumor lesion.

Table 1. Putative Cell Surface Markers of Presumptive TIC

\begin{tabular}{|c|c|}
\hline Tumour Type & Surface Markers \\
\hline Acute Myeloid Leukaemia & $\mathrm{CD}^{2} 4^{+} / \mathrm{CD} 38^{-} / \mathrm{CD} 44^{+} / \mathrm{IL} 3 \mathrm{R}^{+} / \mathrm{CD} 33^{+} / \mathrm{CD} 13^{+}$ \\
\hline Chronic Myeloid Leukemia & $\mathrm{CD} 34^{+} / \mathrm{CD} 38^{-} / \mathrm{CD} 44^{+} / \mathrm{CD} 13^{+}$ \\
\hline $\begin{array}{l}\text { T-cell acute lymphoblastic } \\
\text { leukemia }\end{array}$ & $\mathrm{CD} 34^{+} / \mathrm{CD}^{-} /$or $\mathrm{CD} 34^{+} / \mathrm{CD}^{-}$ \\
\hline Breast carcinoma & $\mathrm{CD} 44^{+} / \mathrm{CD} 24^{-}$ \\
\hline Glioblastoma/medulloblastoma & $\mathrm{CD} 133^{+} /$nestin $^{+} /$Lin $^{-}$ \\
\hline Colon carcinoma & $\mathrm{CD}_{133^{+}}$ \\
\hline Prostate carcinoma & $\mathrm{CD} 133^{+}$? \\
\hline Pancreatic carcinoma & $\mathrm{CD}^{2} 4^{+} / \mathrm{CD} 24^{+} / \mathrm{ESA}^{+}$ \\
\hline Renal carcinoma & $\mathrm{CD}_{133^{+}} ?$ \\
\hline $\begin{array}{l}\text { Squamous cell carcinoma } \\
\text { (head and neck) }\end{array}$ & $\mathrm{CD} 44^{+}$ \\
\hline Melanoma & $\mathrm{CD} 20^{+}$ \\
\hline Neuroblastoma & ABCG2/BCRP1 ${ }^{+}$- Hoescht dye efflux \\
\hline Hepatocellular carcinoma & ABCG2/BCRP1 ${ }^{+}$- Hoescht dye efflux \\
\hline Lung & (Bronchioalveolar cell phenotype) \\
\hline $\begin{array}{l}\text { Various cell line-derived 'side } \\
\text { populations' }\end{array}$ & $\mathrm{ABCG} 2 / \mathrm{BCRP} 1^{+}$- Hoescht dye efflux \\
\hline
\end{tabular}

Culture-selected cells from certain brain tumors exhibit neural stem cell-like multipotency upon proper stimulation (giving rise to neurons and glia) and can effectively be subcloned into highly malignant cell populations in vivo [5-7]. Single cells from neurospheres arising spontaneously in culture from these cells are rather effective in generating multiple secondary clones, but the actual capacity of each of these 
subclones to generate a new tumor in vivo was not documented. These traits, together with some of the tumor-typical gene expression patterns, still rather convincingly unify some consensus properties of neural stem cells and TIC. However, the auto-regenerative, self-renewal potential of these cells and its analogy with normal neural stem cells in terms of Bmi-1-dependency remains elusive [35, 36]. Intriguingly, neural TIC isolated from glioblastoma multiforme maintain their multipotency following neoplastic grow in a rather "protected microenvironment" (i.e. in SCID/bg mice) in vivo, while repeated transplantation apparently augments their malignancy degree [7]. Once again, it is difficult to appreciate how these observations would sustain selfrenewal potential of the cells in vivo, without the formal proof of the persistency of exactly the same genetically tagged cell in these transplantation experiments. It may ultimately be argued that a number of characteristic features of normal stem cells are shared experimentally by subpopulations of cells isolated from tumour lesions, which simultaneously exhibit traits of malignant cells. Other more stringent stem cell traits seem more difficult to demonstrate in laboratory settings, currently leaving the precise identity of these cells established by experimental means open to further definition. Finally, the "clonality" argument for the identification of putative TIC may not be entirely valid. Accumulation of multiple gene mutations may cause phenotypically diverse populations even in cases in which all cells originate from a single clone.

\section{EVIDENCE AND CHARACTERISTICS OF LEUKE- MIA STEM CELLS}

Stem cells from somatic tissues have been best characterized by the haematopoietic system, largely owing to the easy accessibility of blood-forming cells. Hence, it is not surprising that the stem cell malignancies that have been analyzed in the most detail arise in hematologic cancer, leukaemia. With the identification of leukaemia stem cells (LSCs) capable of repopulating NOD/SCID mice, this body of research has led to conclusive proof for TIC.

These are readily evident in chronic myelogenous leukaemia (CML) [37, 38] and acute myelogenous leukaemia (AML) $[39,40]$, and they have been implicated in acute lymphoblastic leukaemia (ALL) [41-43]. The LSCs for human AML were identified as Thy1-/CD34+/CD38- cells that are the sole capable of transferring AML from the human patient to NOD/SCID mice. Several groups have reported that surface markers are differentially expressed in LSCs and normal stem cells. In the case of human AML stem cells, the following series of studies have defined their immunophenotype as CD34+/CD38-/CD71-/HLA-DR-/CD90-/CD117-/ CD123+ (Table 1). The expression of the last 3 antigens in AML stem cells differs from that of normal HSCs, and this difference therefore provides the means for separating normal HSCs from AML stem cells [44]. Perhaps the most promising unique marker of LSCs is CD123 (IL-3R ), because it is present on $98 \%$ of the CD34+/CD38- AML cells, but is undetectable or expressed at lower levels in normal bone marrow CD34+/CD38- cells. Another marker that is detectable on LSCs in patients with AML, but not on normal HSCs is CD33 [45]. This observation is particularly remarkable, given the recent introduction of CD33-targeted therapy (gentuzumab ozogamicin) in clinical trials. Leukaemia stem cells from CML are CD34+/CD38-/CD44+, and TIC in various myeloid neoplasms appear to express the aminopeptidase$\mathrm{N}(\mathrm{CD} 13)$ antigen (Table 1). In addition to the immunophenotype, some studies have examined the cell cycle status of LSCs and found that both AML and CML stem cells maintain quiescent populations. LSCs often exhibit quiescence, which may make the malignant population refractory to standard chemotherapy, or at least no more susceptible than normal HSCs, thus contributing to relapse [46]. Moreover, a recent report [42] has demonstrated the long-term repopulating ability of the CD34+/CD4- and CD34+/CD7- subfractions in T-ALL, suggesting that a cell with a more primitive phenotype was the target for leukaemia transformation in these cases. Finally, mature leukaemia cells might acquire LSC characteristics (Fig. 1D), thereby evading chemotherapeutic treatment and sustaining the disease [46]. Ongoing research is likely to reveal the molecular mechanisms responsible for LSC characteristics and lead to novel strategies for eradicating leukaemia.

\section{CONCLUSIVE CAUTION AND OPTIMISM}

An important aspect of the current debate on "cancer stem cells"/TIC is whether the cells defined as such in both tumor lesions and cultured cell lines are truly self-renewing elements responsible for growth and long-term maintenance of the tumor, or whether they simply represent a particularly aggressive (malignant) side population that has been selected overtime in the lesion to survive the most harsh environmental conditions. If such a subpopulation of tumor cells is largely quiescent, resistant to hypoxia and nutrient deprivation, and has acquired a pronounced apoptosis-/drugresistance, then it is not surprising that upon transplantation it may regenerate a similar tumor lesion. The challenges (i.e. the stimuli) from the new environment into which these cells are transplanted would be ideal for the re-activation of the cell cycle of these cells and for the support of their propagation. Furthermore, if these cells reside originally in a stasismaintaining environment of the lesion, maybe the niche itself, then their removal from such an environment would provide them with the proper stimulation to resume growing. Besides the fact that this phenomenon would not per se be indicative of a canonical self-renewing process, a major concern remains the identification of the exact location of TIC in the tumor lesions in situ.

A related issue is the specificity of the markers that are currently being used to isolate stem-like cells from different solid cancers (Table 1). First, it is v ery unlikely that CD44 (or CD24) would specifically characterize TIC because it is too a widespread cell surface proteoglycan to be restricted to defined, rare subpopulations of tumor cells. It is similarly rather surprising that CD133 emerges as a universal TIC marker across tumors derived from cells of all three embryonic germ layers. "Functional" cell isolation assays based upon dye efflux through drug transporters also seem rather unreliable for the identification of rare subpopulation since through such assays up to $30 \%$ of the tumor cells of the lesion are commonly separated. Such numbers would not be compatible with a rare tumor cell population. Nevertheless, one cannot deny the appeal of explaining the pool of drugresistant cells and the problem of chemotherapy resistance in terms of the existence of a relatively quiescent cell population armed with multiple drug transporters. How then does 
this model fit in the context of the clinical problem of drug resistance? Unfortunately, for most drug-resistant cancers, including melanoma, renal, pancreatic and sarcoma tumors, the hurdle is not that a few cells survive but, rather, that only a few cells die in response to chemotherapy. Due to this vexing problem, the TIC model of drug resistance has little applicability, whereas for tumor relapse following successful therapeutic intervention it could be of greater relevance.

Although the "cancer stem"/TIC case needs to be firmly proven [47], vital information has recently emerged by examining the gene expression profiles of postulated TIC. In breast carcinoma patients these putative TIC-like cells express genes that when evaluated prognostically may have a certain impact on the clinical management of these patients [48]. Similarly, a stem cell-like 11-gene profile down-stream of Bmi-1 proposes a signature predictive of a short interval to disease recurrence, distant metastases, and death after therapy in patients affected by 11 different types of tumors [49]. This latter finding strongly suggest that isolation and phenotypic characterization of putative TIC from different types of tumors could allow for the disclosure of the critical molecular profiles of any given tumor, as well as the exploitation of this genetic information to improve the current diagnostic tools and advance our possibilities of designing novel therapeutic strategies. It is possible that a better understanding of the origin, identity, and traits of particularly malignant subpopulations of cells within lesions may afford valuable information about the intractable nature of many human cancers and explains the reason for which conventional anti-neoplastic therapy fails in many patients.

\section{ACKNOWLEDGEMENT}

We thank Ms. Elena Byther for her expert secretarial assistance.

\section{REFERENCES}

[1] Caussinus E, Gonzalez C. Induction of tumor growth by altered stem-cell asymmetric division in Drosophila melanogaster. Nat Genet 2005; 37: 1125-29.

[2] Pardal R, Clarke MF, Morrison SJ. Applying the principles of stem-cell biology to cancer. Nat Rev Cancer 2003; 3; 895-902.

[3] Jordan CT, Guzman ML, Noble M. Cancer stem cells. N Engl J Med 2006; 355: 1253-61.

[4] Reya T, Morrison SJ, Clarke MF, Weissman IL. Stem cells, cancer, and cancer stem cells. Nature 2001; 414: 105-11.

[5] Singh SK, Hawkins C, Clarke ID, et al. Identification of human brain tumour initiating cells. Nature 2004; 432: 396-01.

[6] Galli R, Binda E, Orfanelli U, et al. Isolation and characterization of tumorigenic, stem-like neural precursors from human glioblastoma. Cancer Res 2004; 64: 7011-21.

[7] Vescovi AL, Galli R, Reynolds BA. Brain tumour stem cells. Nat Rev Cancer 2006; 6: 425-36.

[8] Al-Hajj M, Wicha MS, Benito-Hernandez A, Morrison SJ, Clarke MF. Prospective identification of tumorigenic breast cancer cells. Proc Natl Acad Sci USA 2003; 100: 3983-88.

[9] Tang DG, Patrawala L, Calhoun T, et al. Prostate cancer stem/progenitor cells: identification, characterization, and implications. Mol Carcinog 2007; 46: 1-14.

[10] Prince ME, Sivanandan R, Kaczorowski A, et al. Identification of a subpopulation of cells with cancer stem cell properties in head and neck squamous cell carcinoma. Proc Natl Acad Sci USA 2007; 104: 973-78.

[11] Li C, Heidt DG, Dalerba P, et al. Identification of pancreatic cancer stem cells. Cancer Res 2007; 67: 1030-37.

[12] Kim CF, Jackson EL, Woolfenden AE, et al. Identification of bronchioalveolar stem cells in normal lung and lung cancer. Cell 2006; 121: 823-35.
[13] Chiba T, Kita K, Zheng YW, et al. Side population purified from hepatocellular carcinoma cells harbors cancer stem cell-like properties. Hepatology 2006; 44: 240-51.

[14] Fang D, Nguyen TK, Leishear K, et al. A tumorigenic subpopulation with stem cell properties in melanoma. Cancer Res 2005; 65: 9328-37.

[15] O'Brien CA, Pollett A, Galliger S, Dick JE. A human colon cancer cell capable of initiating tumour growth in immunodeficient mice. Nature 2007; 445: 106-10.

[16] Ricci-Vitiani L, Lombardi DG, Pilozzi E, et al. Identification and expansion of human colon-cancer-initiating cells. Nature 2007; 445: 111-15.

[17] Hirschmann-Jax C, Foster AE, Wulf GG, et al. A distinct 'side population' of cells with high drug efflux capacity in human tumor cells. Proc Natl Acad Sci USA 2004; 101: 14228-33.

[18] Heppner GH, Miller FR. The cellular basis of tumor progression. Int Rev Cytol 1998; 177: 1-56.

[19] Hanahan D, Weinberg RA. The hallmarks of cancer. Cell 2000; 100: $57-70$.

[20] Marx J. Cancer research. Mutant stem cells may seed cancer. Science 2003; 301: 1308-10.

[21] Gazziola C, Cordani N, Colombatti A, Perris R. Malignant fibrous histiocytoma: a proposed cellular origin and identification of its characterizing gene transcripts. Int J Oncol 2003; 23: 343-51.

[22] Riggi N, Cironi L, Provero P, et al. Development of Ewing's sarcoma from primary bone marrow-derived mesenchymal progenitor cells. Cancer Res 2005; 65: 11459-68.

[23] Houghton J, Stoicov C, Nomura S, et al. Gastric cancer originating from bone marrow-derived cells. Science 2004; 306: 1568-71.

[24] Handgretinger R, Gordon PR, Leimig T, et al. Biology and plasticity of CD $133^{+}$hematopoietic stem cells. Ann NY Acad Sci 2003; 996: 141-51.

[25] Bussolati B, Bruno S, Grange C, et al. Isolation of remnal progenitor cells from adult human kidney. AM J Pathol 2005; 166: 545-55.

[26] Locke M, Heywood M, Fawell S, Mackenzie IC. Retention of intrinsic stem cell hierarchies in carcinoma-derived cell lines. Cancer Res 2005; 65: 8944-50.

[27] Ilyas M, Straub J, Tomlinson IP, Bodmer WF. Genetic pathways in colorectal and other cancers. Eur J Cancer 1999; 35: 1986-2002.

[28] Van Deursen JM. Rb loss causes cancer by driving mitosis mad. Cancer Cell 2007; 11: 1-3.

[29] Feinberg AP, Ohlsson R, Henikoff S. The epigenetic progenitor origin of human cancer. Nat Rev Genet 2006; 7: 21-33.

[30] Li L, Xie T. Stem cell niche: structure and function. Annu Rev Cell Dev Biol 2005; 21: 605-31.

[31] Haramis AP, Begthel H, van den Born M, et al. De novo crypt formation and juvenile polyposis on BMP inhibition in mouse intestine. Science 2004; 303: 1684-86.

[32] Li L, Neaves WB. Normal stem cells and cancer stem cells: the niche matters. Cancer Res 2006; 66: 4553-57.

[33] Calabrese C, Poppleton H, Kocak M, et al. A perivascular niche for brain tumor stem cells. Cancer Cell 2007; 11: 69-82.

[34] Krause DS, Theise ND, Collector MI, et al. Multi-organ, multilineage engraftment by a single bone marrow-derived stem cell. Cell 2001; 105: 369-77.

[35] Molofsky AV, Pardal R, Iwashita T, Park IK, Clarke MF. Morrison SJ. Bmi-1 dependence distinguishes neural stem cell self-renewal from progenitor proliferation. Nature 2003; 425: 962-67.

[36] Liu S, Dontu G, Mantle ID, et al. Hedgehog signaling and Bmi-1 regulate self-renewal of normal and malignant human mammary stem cells. Cancer Res 2006; 66: 6063-71.

[37] Holyoake TL, Jiang X, Drummond MW, Eaves AC, Eaves CJ. Elucidating critical mechanisms of deregulated stem cell turnover in the chronic phase of chronic myeloid leukemia. Leukemia 2002; 16: 549-58.

[38] Florian S, Sonneck K, Hauswirth AW, et al. Detection of molecular targets on the surface of CD34+/CD38-- stem cells in various myeloid malignancies. Leuk Lymphoma 2006; 47: 207-22.

[39] Lapidot T, Sirard C, Vormoor J, et al. A cell initiating human acute myeloid leu-kaemia after transplantation into SCID mice. Nature 1994; 367: 645-48.

[40] Bonnet D, Dick JE. Human acute myeloid leukemia is organized as a hierarchy that originates from a primitive hematopoietic cell. Nat Med 1997; 3: 730-37. 
[41] Castor A, Nilsson L, Astrand-Grundstrom I, et al. Distinct patterns of hematopoietic stem cell involvement in acute lymphoblastic leukaemia. Nat Med 2005; 11: 630-37.

[42] Cox CV, Martin HM, Kearns PR, Virgo P, Evely RS, Blair A. Characterization of a progenitor cell population in childhood T-cell acute lymphoblastic leukaemia. Blood 2007; 109: 674-82.

[43] Cobaleda C, Gutierrez-Cianca N, Perez- Losada Flores T, GarciaSanz R, Gonzalez M, Sanchez-Garcia I. A primitive hematopoietic cell is the target for the leukemic transformation in human philadelphia-positive acute lymphoblastic leukaemia. Blood 2000; 95: 1007-13.

[44] Hanahan D, Weinberg RA. The hallmarks of cancer. Cell 2000; 100: $57-70$.
[45] Hauswirth AW, Florian S, Printz D, et al. Expression of the target receptor CD33 in CD34/CD38/CD123 AML stem cells. Eur J Clin Invest 2007; 37: 73-82.

[46] Ravandi F, Estrov Z. Eradication of leukemia stem cells as a new goal of therapy in leukaemia. Clin Cancer Res 2006; 12: 340-44.

[47] Hill RP. Identifying cancer stem cells in solid tumors: case not proven. Cancer Res 2006; 66: 1891-95.

[48] Liu R, Wang X, Chen GY, et al. The prognostic role of a gene signature from tumorigenic breast-cancer cells. N Engl J Med 2007; 356: 217-26.

[49] Glinsky GV, Berezovska O, Glinskii AB. Microarray analysis identifies a death-from-cancer signature predicting therapy failure in patients with multiple types of cancer. J Clin Invest 2005; 115: 1503-21. 